Cite this article as: BMJ, doi:10.1136/bmj.38993.731725.BE (published 10 November 2006) Research

\title{
Lifetime cost effectiveness of simvastatin in a range of risk groups and age groups derived from a randomised trial of 20536 people
}

Heart Protection Study Collaborative Group

\begin{abstract}
Objectives To evaluate the cost effectiveness of $40 \mathrm{mg}$ simvastatin daily continued for life in people of different ages with differing risks of vascular disease.

Design A model developed from a randomised trial was used to estimate lifetime risks of vascular events and costs of treatment and hospital admissions in the United Kingdom. Setting 69 hospitals in the UK.

Participants 20536 men and women (aged 40-80) with coronary disease, other occlusive arterial disease, or diabetes. Interventions $40 \mathrm{mg}$ simvastatin daily versus placebo for an average of 5 years.

Main outcome measures Cost effectiveness of $40 \mathrm{mg}$ simvastatin daily expressed as additional cost per life year gained. Major vascular event defined as non-fatal myocardial infarction or death from coronary disease, any stroke, or revascularisation procedure. Results were extrapolated to younger and older age groups at lower risk of vascular disease than were studied directly, as well as to lifetime treatment. Results At the April 2005 UK price of $£ 4.87$ (€7; \$9) per 28 day pack of generic $40 \mathrm{mg}$ simvastatin, lifetime treatment was cost saving in most age groups and vascular disease risk groups studied directly. Gains in life expectancy and cost savings decreased with increasing age and with decreasing risk of vascular disease. People aged 40-49 with 5 year risks of major vascular events of $42 \%$ and $12 \%$ at start of treatment gained 2.49 and 1.67 life years, respectively. Treatment with statins remained cost saving or cost less than $£ 2500$ per life year gained in people as young as 35 years or as old as 85 with 5 year risks of a major vascular event as low as $5 \%$ at the start of treatment.

Conclusions Treatment with statins is cost effective in a wider population than is routinely treated at present.
\end{abstract}

\section{Introduction}

Large randomised trials have shown that lowering blood concentrations of low density lipoprotein cholesterol with statins greatly reduces rates of heart attacks, strokes, and revascularisation procedures in a wide range of people at high risk, largely irrespective of their cholesterol concentrations and other characteristics at presentation. ${ }^{1}$ The heart protection study has shown that, especially when cheaper generic versions are used, $40 \mathrm{mg}$ simvastatin daily is cost effective for a wider range of people with vascular disease or diabetes than previously thought. ${ }^{2}$ That analysis only estimated cost effectiveness during the study treatment period and did not provide estimates of the additional costs per life year or quality adjusted life year gained. ${ }^{3}$
The present report extends this previous work in two ways. Firstly, a model was developed and validated that allowed extrapolation beyond the five year treatment period in the heart protection study and estimation of lifetime cost per life year gained and per quality adjusted life year gained; this facilitates comparison with other potential uses of healthcare resources. Secondly, these estimates were projected beyond the levels of risk of vascular disease and age represented in the study to provide guidance on the cost effectiveness of statins in a wider spectrum of people.

\section{Methods}

We used data from the 20536 participants in the heart protection study to develop a Markov disease state transition model. Participants were men and women who presented at 40-80 years with total cholesterol concentrations of at least 3.5 $\mathrm{mmol} / \mathrm{l}(135 \mathrm{mg} / \mathrm{dl})$ and a medical history of coronary disease, cerebrovascular disease, other occlusive arterial disease, diabetes mellitus, or (if a man aged $\geq 65$ ) treated hypertension. ${ }^{4}$ Figure 1 is a schematic representation of the model, which is based around the prediction of four key events. The annual risks of occurrence of three main adverse events ("death from vascular cause" defined as death from coronary disease, fatal stroke, or other vascular event; "non-fatal major vascular event" defined as non-fatal myocardial infarction, non-fatal stroke, or arterial revascularisation; and "other vascular event" defined as admission for angina, heart failure, or other cardiac or vascular problem) were estimated as functions of baseline risk factors, allocation to statin treatment, and within-study experience of vascular events. During the course of the heart protection study, some participants allocated simvastatin stopped taking statin therapy ( $18 \%$ by the end of the study) and some participants allocated placebo started taking non-study statin $(32 \%$ by the end). ${ }^{4}$ To assess the full effects of actually taking simvastatin, the risk equations in the model incorporated a factor for the proportion of full compliance seen between the treatment arms. The effect of statin therapy used in the model was specific to each vascular event category, with the same effect fitted for first and subsequent events in the same category. We estimated the fourth event in the model (mortality rate for non-vascular causes) by removing deaths due to vascular causes from official life table data for the United Kingdom. ${ }^{5-7}$ Allocation to $40 \mathrm{mg}$ simvastatin daily was not associated with any significant excess of reported muscle symptoms (including a non-significant annual

A table, a technical appendix, and details of collaborators, participating hospitals, and committees are on bmj.com 


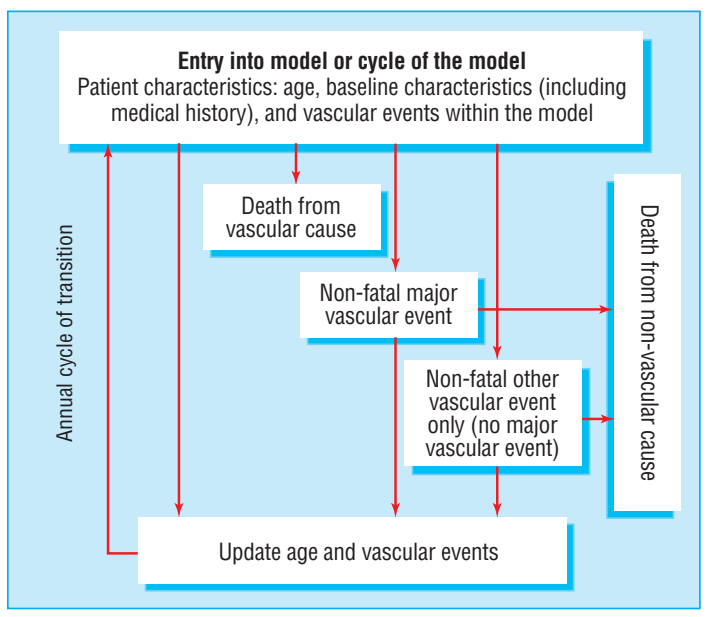

Fig 1 Schematic of the state transition model

excess myopathy rate of $0.01 \% ; \mathrm{P}=0.2$ ) or of other adverse events. ${ }^{4}$ Thus, such adverse events were not included in the current analyses.

We used the model to predict the annual occurrence of death due to vascular or non-vascular causes; of non-fatal major vascular events; or of other vascular events and the annual costs of hospital admissions for such events in people taking or not taking $40 \mathrm{mg}$ simvastatin daily. Annual costs of hospital admissions (2001 UK prices) were estimated from data on hospital admission in the heart protection study ${ }^{2}$ on the basis of age, sex, disease history, other baseline characteristics, and vascular events or death within the study. Treatment affected the risk of an event occurring, but we found no evidence that it affected costs of hospital admissions once the event had occurred. Annual rates of vascular events predicted by the model were internally validated against the rates seen during the five year follow-up in the heart protection study for the proportion of participants taking statin in each group and the average difference in low density lipoprotein cholesterol seen during the study. The model was then used to project events and costs beyond the five year scheduled treatment period and to estimate lifetime cost effectiveness of $40 \mathrm{mg}$ simvastatin daily versus no statin treatment among people of different ages and with different underlying risks of vascular events. In the main analysis, we used the UK tariff price of $£ 4.87$ (€7;\$9) at which pharmacies were reimbursed for 28 days of treatment in April 2005. ${ }^{8}$ Future life years and costs were discounted at an annual rate of $3.5 \%{ }^{9}$

As in previous analyses, ${ }^{2}$ participants were divided into five similar sized groups of estimated five year risk of a major vascular event, with average risks in the groups ranging from $12 \%$ to $42 \%$ (which correspond to risks of $4 \%$ to $12 \%$ for non-fatal myocardial infarction or coronary death). These risk groups were subdivided by age at entry to the study (40-49, 50-59, 60-69, and $\geq 70$ ) to allow for the opposing effects of age on disease risk and life expectancy. Parameter uncertainty in the estimates of life

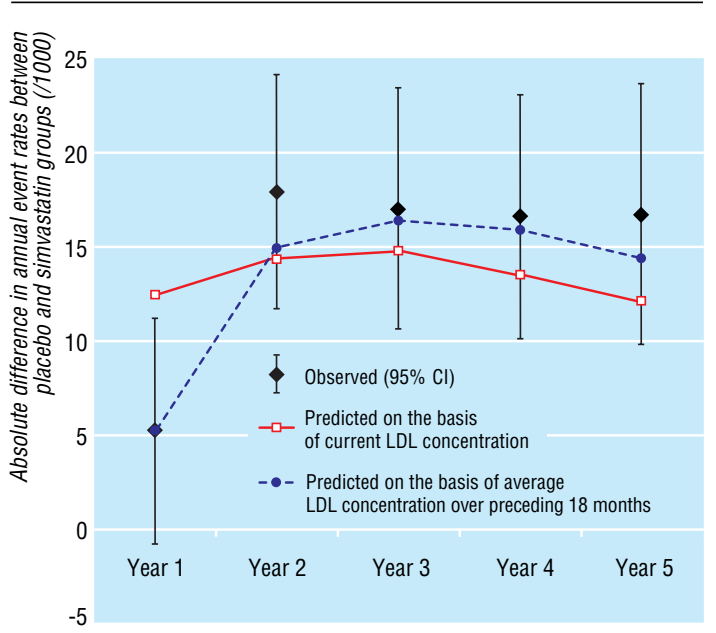

Fig 2 Observed (95\% confidence interval) and predicted difference in annual rates of major vascular events or deaths from vascular causes between groups allocated to placebo and simvastatin in the heart protection study. LDL=Iow density lipoprotein

years gained, cost savings in hospital stay, and cost per life year gained was assessed by non-parametric bootstrapping. ${ }^{10}$ We also examined several other scenarios. Firstly, predicted life expectancy was adjusted for age specific and sex specific health related quality of life derived from a representative sample of the UK population ${ }^{11}{ }^{12}$; secondly, it was related to only five years' use of generic simvastatin; and thirdly, it was related to lifetime use of proprietary simvastatin (2005 UK price of $£ 29.69$ for 28 days)..$^{13}$ Finally, the model was extrapolated to older and younger age groups and to people at lower risk of vascular disease than those included in the heart protection study. Age at start of treatment was projected to five years beyond the eligibility limits of the study (down to 35 and up to 85), and risk was projected down to a $5 \%$ risk of a major vascular event within five years (compared with the $12 \%$ five year risk in the fifth with the lowest risk in the heart protection study). See bmj.com for a technical appendix containing additional details of the methods and model validation, along with a further scenario related to diminishing long term compliance with treatment.

\section{Results}

\section{Internal validation of the Markov state transition model}

Annual rates of death from vascular disease, of major vascular events, and of all vascular events estimated from the state transition model (fig 1) were similar to those seen during the heart protection study, as were the differences in rates (see technical appendix on bmj.com). Figure 2 shows the similarity between predicted and observed differences in the annual rates of first and subsequent non-fatal major vascular events or deaths from vascular disease during the five year treatment period (especially

Table 1 Predicted life years gained (95\% confidence interval) by full compliance with lifetime use of 40 mg simvastatin daily for the population of the heart protection study (not discounted)

\begin{tabular}{|c|c|c|c|c|c|}
\hline \multirow{2}{*}{ Age at start (years) } & \multicolumn{5}{|c|}{ Five year risk of major vascular event at start of treatment } \\
\hline & $12 \%$ & $18 \%$ & $22 \%$ & $28 \%$ & $42 \%$ \\
\hline $40-49$ & 1.67 (0.84 to 2.47$)$ & $2.02(1.13$ to 2.85$)$ & 2.21 (1.27 to 3.07$)$ & 2.41 (1.45 to 3.26) & 2.49 (1.55 to 3.36$)$ \\
\hline $50-59$ & 1.32 (0.66 to 2.00$)$ & $1.52(0.82$ to 2.22$)$ & 1.67 (0.94 to 2.40$)$ & 1.84 (1.07 to 2.60$)$ & 1.94 (1.18 to 2.68$)$ \\
\hline $60-69$ & $0.95(0.47$ to 1.47$)$ & $1.06(0.55$ to 1.62$)$ & $1.17(0.63$ to 1.74$)$ & 1.27 (0.71 to 1.86$)$ & $1.39(0.82$ to 1.99$)$ \\
\hline$\overline{\geq 70}$ & 0.64 (0.31 to 0.98$)$ & $0.72(0.36$ to 1.11$)$ & 0.79 (0.41 to 1.19$)$ & 0.88 (0.48 to 1.29$)$ & 0.98 (0.56 to 1.39$)$ \\
\hline
\end{tabular}


Table 2 Outcome measures with full compliance with lifetime use of generic $40 \mathrm{mg}$ simvastatin daily for the population of the heart protection study

\begin{tabular}{llllll} 
& \multicolumn{6}{c}{ Five year risk of major vascular event at start of treatment } \\
\cline { 2 - 6 } Outcome & $\mathbf{1 2 \%}$ & $\mathbf{1 8 \%}$ & $\mathbf{2 2} \%$ & $\mathbf{2 8} \%$ & $\mathbf{4 2} \%$ \\
\hline $\begin{array}{l}\text { Life years gained } \\
\begin{array}{l}\text { Age at start } \\
\text { (years): }\end{array}\end{array}$ & & & & & \\
\hline $40-49$ & 0.62 & 0.81 & 0.94 & 1.08 & 1.24 \\
\hline $50-59$ & 0.58 & 0.71 & 0.82 & 0.94 & 1.08 \\
\hline $60-69$ & 0.50 & 0.58 & 0.66 & 0.75 & 0.88 \\
\hline$\geq 70$ & 0.38 & 0.45 & 0.50 & 0.57 & 0.68 \\
\hline
\end{tabular}

Lifetime cost (£) of treatment with simvastatin

Age at start

\begin{tabular}{lccccc} 
(years): & \multicolumn{10}{l}{} & & & \\
\hline $40-49$ & 1180 & 1100 & 1050 & 990 & 880 \\
\hline $50-59$ & 1030 & 950 & 900 & 860 & 760 \\
\hline $60-69$ & 840 & 780 & 730 & 680 & 590 \\
\hline$\geq 70$ & 670 & 620 & 580 & 540 & 460
\end{tabular}

Cost (£) of hospital admission due to simvastatin*

Age at start

\begin{tabular}{lccccc} 
(years): & & & & & \\
\hline $40-49$ & -1450 & -1810 & -1970 & -2080 & -2100 \\
\hline $50-59$ & -1250 & -1400 & -1460 & -1570 & -1530 \\
\hline $60-69$ & -920 & -980 & -990 & -1010 & -950 \\
\hline$\geq 70$ & -640 & -650 & -660 & -650 & -560
\end{tabular}

Cost (£) per life year gained

Age at start

(years):

\begin{tabular}{lccccc}
\hline $40-49$ & -440 & -880 & -990 & -1010 & -990 \\
\hline $50-59$ & -370 & -630 & -680 & -760 & -720 \\
\hline $60-69$ & -140 & -360 & -400 & -440 & -400 \\
\hline$\geq 70$ & 80 & -70 & -160 & -190 & -140
\end{tabular}

Negative values indicate cost savings. $£ 1=€ 1.5=\$ 1.9$.

All results discounted at $3.5 \%$ per annum; 28 day pack of generic simvastatin at $£ 4.87$.

when the average low density lipoprotein cholesterol difference during the preceding 18 months was used in the model).

Cost effectiveness of simvastatin for the study population

We used the model to estimate the life expectancy and costs associated with lifetime treatment with $40 \mathrm{mg}$ simvastatin daily. On the basis of full compliance, relative reductions in the risk of death from vascular disease by $25 \%$, of a non-fatal major vascular event or vascular death by $32 \%$, and of any vascular event by $24 \%$ were derived from the risk equations. Table 1 shows the undiscounted estimated gains in life expectancy among the participants subdivided into fifths of five year risk of a major vascular event and by age at the start of treatment. The estimated life years gained ranged between 0.64 years for people aged over 70 with a $12 \%$ five year risk of a major vascular event and 2.49 years for those aged $40-49$ with a $42 \%$ five year risk. Table 2 shows the estimates for discounted life years gained, costs of statin treatment, cost savings for hospital admissions, and costs per life year gained. At $£ 4.87$ per 28 day pack of treatment, generic 40 $\mathrm{mg}$ simvastatin daily was cost saving for most of the risk and age categories in the heart protection study; that is, the reduced costs of hospital admissions as a result of fewer vascular events outweighed the increased costs of statin treatment in all but one category ( $£ 80$ per life year gained for people aged $\geq 70$ years at the start with a $12 \%$ five year risk; table 2 ). The upper limits of the $95 \%$ confidence intervals for all risk and age groups were below $£ 1000$ per life year gained (table A on bmj.com).

The heart protection study did not collect information on quality of life, so evaluation of cost per quality adjusted life year gained involved using data from a representative sample of the UK population to adjust for age specific and sex specific health related quality of life. ${ }^{11}{ }^{12}$ Quality of life adjusted estimates for lifetime use of generic $40 \mathrm{mg}$ simvastatin daily were similar to the unadjusted estimates (table 3). Estimated costs per life year gained with only five years' use of generic $40 \mathrm{mg}$ simvastatin daily were also similar to those for lifetime treatment, with both costs and benefits reduced proportionally (table 4). Cost effectiveness estimates were favourable even with proprietary 40 mg simvastatin at £29.69 per 28 day pack (table 4).

Table 3 Additional scenarios of full compliance with $40 \mathrm{mg}$ simvastatin daily within the population of the heart protection study. Values are life years gained unless stated otherwise

\begin{tabular}{lccccc} 
& \multicolumn{5}{c}{ Five year risk of major vascular event at start of treatment } \\
\cline { 2 - 6 } Treatment & $\mathbf{1 2 \%}$ & $\mathbf{1 8 \%}$ & $\mathbf{2 2} \%$ & $\mathbf{2 8 \%}$ & $\mathbf{4 2} \%$ \\
\hline Lifetime use of generic simvastatin* & & & & \\
\hline Age at start (years): & & & & & \\
\hline $40-49$ & 0.47 & 0.63 & 0.72 & 0.84 & 0.97 \\
\hline $50-59$ & 0.43 & 0.54 & 0.63 & 0.72 & 0.83 \\
\hline $60-69$ & 0.36 & 0.43 & 0.50 & 0.56 & 0.66 \\
\hline$\geq 70$ & 0.27 & 0.33 & 0.37 & 0.43 & 0.51 \\
\hline
\end{tabular}

5 year use of generic simvastatin

Age at start (years):

\begin{tabular}{llllll}
\hline $40-49$ & 0.09 & 0.13 & 0.17 & 0.21 & 0.29 \\
\hline $50-59$ & 0.11 & 0.15 & 0.19 & 0.23 & 0.32 \\
\hline $60-69$ & 0.13 & 0.17 & 0.20 & 0.25 & 0.35 \\
\hline$\geq 70$ & 0.14 & 0.17 & 0.20 & 0.24 & 0.34
\end{tabular}

Lifetime use of proprietary simvastatin

Age at start (years):

\begin{tabular}{llllll}
\hline $40-49$ & 0.62 & 0.81 & 0.94 & 1.08 & 1.24 \\
\hline $50-59$ & 0.58 & 0.71 & 0.82 & 0.94 & 1.08 \\
\hline $60-69$ & 0.50 & 0.58 & 0.66 & 0.75 & 0.88 \\
\hline$\geq 70$ & 0.38 & 0.45 & 0.50 & 0.57 & 0.68
\end{tabular}

*Values are quality adjusted life years gained (adjusted for age specific and sex specific health related quality of life).

All results discounted at $3.5 \%$ per annum.

Table 4 Cost (£) per life year gained (unless stated otherwise) for various scenarios of full compliance with $40 \mathrm{mg}$ simvastatin daily for the population of the heart protection study

\begin{tabular}{|c|c|c|c|c|c|}
\hline \multirow[b]{2}{*}{ Treatment } & \multicolumn{5}{|c|}{ Five year risk of major vascular event at start of treatment } \\
\hline & $12 \%$ & $18 \%$ & $22 \%$ & $28 \%$ & $42 \%$ \\
\hline \multicolumn{6}{|c|}{ Lifetime use of generic simvastatin* } \\
\hline \multicolumn{6}{|c|}{ Age at start (years): } \\
\hline 40-49 & -580 & -1140 & -1280 & -1310 & -1270 \\
\hline $50-59$ & -500 & -830 & -890 & -990 & -930 \\
\hline $60-69$ & -200 & -480 & -530 & -580 & -530 \\
\hline$\geq 70$ & 110 & -100 & -220 & -250 & -190 \\
\hline
\end{tabular}

5 year use of generic simvastatin

Age at start (years):

\begin{tabular}{lccccc}
\hline $40-49$ & -1230 & -1890 & -2030 & -2050 & -1930 \\
\hline $50-59$ & -1010 & -1330 & -1380 & -1470 & -1350 \\
\hline $60-69$ & -600 & -840 & -870 & -900 & -800 \\
\hline$\geq 70$ & -240 & -410 & -500 & -520 & -410
\end{tabular}

Lifetime use of proprietary simvastatin

Age at start (years)

\begin{tabular}{llllll}
\hline Age at start (years): & & & & & \\
\hline $40-49$ & 9260 & 6010 & 4710 & 3620 & 2610 \\
\hline $50-59$ & 8720 & 6170 & 4900 & 3870 & 2860 \\
\hline $60-69$ & 8470 & 6430 & 5230 & 4190 & 3040 \\
\hline$\geq 70$ & 8910 & 6910 & 5740 & 4610 & 3340 \\
\hline
\end{tabular}

*Values are adjusted for age specific and sex specific health related quality of life. Negative values indicate cost savings. $£ 1=€ 1.5=\$ 1.9$.

All discounted at $3.5 \%$ per annum; 28 day pack of generic simvastatin at $£ 4.87$ and proprietary simvastatin at $£ 29.69$. 
Table 5 Life years gained by full compliance with lifetime use of generic 40 $\mathrm{mg}$ simvastatin daily projected beyond the population of the heart protection study

\begin{tabular}{|c|c|c|c|c|}
\hline \multirow[b]{2}{*}{ Outcome } & \multicolumn{4}{|c|}{$\begin{array}{c}\text { Five year risk of major vascular event at start of } \\
\text { treatment }\end{array}$} \\
\hline & $5 \%$ & $10 \%$ & $20 \%$ & $40 \%$ \\
\hline \multicolumn{5}{|c|}{ Life years gained } \\
\hline \multicolumn{5}{|c|}{ Age at start (years): } \\
\hline 35 & $0.45^{*}$ & $0.62^{*}$ & $1.02^{*}$ & $1.52^{*}$ \\
\hline 45 & $0.44^{*}$ & 0.58 & 0.90 & 1.34 \\
\hline 55 & $0.41^{*}$ & 0.52 & 0.78 & 1.15 \\
\hline 65 & $0.34^{*}$ & 0.43 & 0.62 & 0.92 \\
\hline 75 & $0.24^{*}$ & 0.31 & 0.43 & 0.63 \\
\hline 85 & $0.14^{*}$ & $0.17^{*}$ & $0.25^{*}$ & $0.35^{\star}$ \\
\hline \multicolumn{5}{|c|}{ Quality adjusted life years gained $\dagger$} \\
\hline \multicolumn{5}{|c|}{ Age at start (years): } \\
\hline 35 & $0.35^{*}$ & $0.48^{*}$ & $0.80^{*}$ & $1.19^{\star}$ \\
\hline 45 & $0.33^{*}$ & 0.44 & 0.70 & 1.04 \\
\hline 55 & $0.30^{*}$ & 0.39 & 0.59 & 0.89 \\
\hline 65 & $0.24^{*}$ & 0.31 & 0.46 & 0.70 \\
\hline 75 & $0.17^{\star}$ & 0.21 & 0.32 & 0.47 \\
\hline 85 & $0.09^{*}$ & $0.12^{*}$ & $0.18^{*}$ & $0.26^{*}$ \\
\hline
\end{tabular}

${ }^{*}$ Categories of patients not generally represented in the heart protection study. †Values adjusted for age specific and sex specific health related quality of life. All results discounted at $3.5 \%$ per annum.

\section{Cost effectiveness of simvastatin beyond the study population}

The heart protection study comprised people presenting at 40-80 years with five year risks of a major vascular event in five similar sized categories ranging from $12 \%$ to $42 \%$. Extrapolations indicate that lifetime use of generic $40 \mathrm{mg}$ simvastatin daily is cost effective for people below and above this age range with five year risk of a major vascular event as low as $5 \%$ (tables 5 and 6 ). The estimated costs per life year gained ranged from about $£ 450$ to $£ 2500$ for people with a $5 \%$ five year risk of a major vascular event aged between 35 and 85 at the start of treatment. Adjusting for age specific and sex specific quality of life did not affect these conclusions; the cost per quality adjusted life year gained was either a saving or less than $£ 4000$ across the

Table 6 Cost effectiveness of full compliance with lifetime use of generic 40 $\mathrm{mg}$ simvastatin daily projected beyond the population of the heart protection study

\begin{tabular}{lcccc} 
& \multicolumn{4}{c}{ Five year risk of major vascular event at start of } \\
treatment
\end{tabular}

Cost (£) per quality adjusted† life year gained

\begin{tabular}{lcccc}
\hline Age at start (years): & & & & \\
\hline 35 & $580^{\star}$ & $-460^{*}$ & $-1370^{*}$ & $-2060^{*}$ \\
\hline 45 & $430^{\star}$ & -480 & -1210 & -1600 \\
\hline 55 & $550^{\star}$ & -280 & -900 & -1070 \\
\hline 65 & $930^{*}$ & 70 & -510 & -590 \\
\hline 75 & $1740^{*}$ & 650 & -50 & -140 \\
\hline 85 & $3740^{*}$ & $1870^{*}$ & $690^{\star}$ & $420^{*}$ \\
\hline
\end{tabular}

${ }^{*}$ Categories of patient not generally represented in the heart protection study. tAdjusted for age specific and sex specific health related quality of life. Negative figures indicate cost savings. $£ 1=€ 1.5=\$ 1.9$.

Discounted at $3.5 \%$ per annum; generic simvastatin at $£ 4.87$ per 28 day pack. extended age groups and risk categories for vascular events considered (table 6).

\section{Discussion}

As well as preventing heart attacks and coronary death, statins have been shown to reduce the incidence of ischaemic strokes, and arterial revascularisation procedures. ${ }^{14}{ }^{14}$ Recent guidelines, therefore, recommend statins for patients at risk of all such major vascular events, not just coronary events. ${ }^{1516}$ In the UK, statins are recommended for people with an estimated 10 year risk of a cardiovascular event of $20 \%$ or more. ${ }^{17}{ }^{18}$ But a large meta-analysis has shown that similar relative reductions in risk are produced by lowering low density lipoprotein cholesterol with statins by the same absolute amount in higher and lower risk populations. ${ }^{1}$ The present analyses indicate that, at current generic prices, the initiation of lifetime treatment with simvastatin is cost saving or very cost effective for people aged 35-85 with risks of major vascular events as low as 1\% per annum (that is, about half the risk threshold proposed by the National Institute for Health and Clinical Excellence; NICE). The estimated costs per life year gained in all these people were lower than those generally considered by NICE to be a cost effective use of health resources. ${ }^{19}$

The heart protection study did not collect data on the quality of life of participants. But, after adjusting for age specific and sex specific health related quality of life derived from the UK population, the estimated costs per quality adjusted life year from lifelong treatment with generic simvastatin remained highly favourable across the extended age and risk groups considered. Sensitivity analyses also indicated that lifetime and five year treatment with generic simvastatin would be similarly cost effective for the populations studied. Lifelong use in these groups even seemed to be cost effective at the much higher UK proprietary price; these results are relevant to countries where simvastatin is more expensive. Although hospital outpatient costs and primary care costs were not measured, including such costs would be unlikely to alter the main conclusions of our analysis given their comparatively low level. ${ }^{20}$ Muscle symptoms were systematically sought in the heart protection study; compared with placebo, allocation to $40 \mathrm{mg}$ simvastatin daily was not associated with significantly more reports of muscle symptoms or, more seriously, more cases of myopathy or rhabdomyolysis. ${ }^{4}$ Although patients and doctors need to be aware that serious muscle problems can occur rarely, ${ }^{21}$ this does not materially affect the cost effectiveness of routine statin use.

The lifetime extrapolation was based on a model developed by using individual participant data from the five year treatment period of the heart protection study. The vascular event rates predicted by the model were comparable to the rates seen during the within-study period. Similar reductions in relative risk with statin therapy were modelled over time, including beyond the study treatment period, because currently available evidence shows that the relative effects are similar in different age groups and during each successive year of treatment, without evidence that the benefits are lost when treatment stops. ${ }^{12}$

\section{Conclusion}

At current UK prices for generic simvastatin, $40 \mathrm{mg}$ simvastatin daily is likely to be cost saving, or would cost less than $£ 2500$ per life year gained, for people with an annual risk of major vascular events of $1 \%$ or more, independently of their age at the start of treatment. Hence, statin therapy should be considered routinely 


\section{What is already known on this topic}

Statins reduce major vascular events, largely independent of a person's cholesterol concentrations at presentation

Overall risk of vascular disease events (not single risk factors) determines the absolute benefits of statin therapy

What this study adds

Statins are cost effective well beyond the treatment thresholds proposed in current UK clinical guidelines

for people across a wider age range and at lower risk of vascular disease than is currently the case.

Most importantly we thank the participants in the heart protection study, as well as the doctors, nurses, and other staff in hospitals and general practices throughout the UK who assisted with its conduct (see bmj.com for details). Contributors: The writing committee comprised Borislava Mihaylova, Andrew Briggs, Jane Armitage, Sarah Parish, Alastair Gray, and Rory Collins. BM helped draft the manuscript and prepare, analyse, validate, and interpret data. JA, SP, and RC helped design and conduct the heart protection study on which these analyses were based. All authors helped design the analyses, interpret results, and draft the manuscript. AG and RC are guarantors. See bmj.com for other contributors.

Funding: UK Medical Research Council, British Heart Foundation, Merck \& Co (manufacturer of simvastatin), and Roche Vitamins Ltd (manufacturer of the vitamins). The study was designed, conducted, analysed, and interpreted independently of all funding sources.

Competing interests: The Clinical Trial Service Unit, University of Oxford (JA, SP, RC), does not accept honoraria or other payments from the drug industry, except for reimbursement of costs to participate in scientific meetings. Staff in the Health Economics Research Centre, University of Oxford (BM, AG), and Section of Public Health and Health Policy, University of Glasgow (AB), occasionally act as paid consultants to the drug industry. The Clinical Trial Service Unit and the Health Economics Research Centre have received research funding through the University of Oxford from Merck \& Co and Roche Vitamins Ltd.

Ethical approval: Local ethics committee approval for each of the 69 participating hospitals.

1 Cholesterol Treatment Trialists' (CTT) Collaborators. Efficacy and safety of cholesterol-lowering treatment: prospective meta-analysis of data from 90056 participants in 14 randomised trials of statins. Lancet 2005;366:1267-78.
2 Heart Protection Study Collaborative Group. Cost-effectiveness of simvastatin in people at different levels of vascular disease risk: economic analysis of a randomised trial in 20536 individuals. Lancet 2005;365:1779-85.

3 Imai K, Zhang P. Integrating economic analysis into clinical trials. Lancet 2005;365:1749-50.

4 Heart Protection Study Collaborative Group. MRC/BHF heart protection study of cholesterol lowering with simvastatin in 20,536 high-risk individuals: a randomised placebo-controlled trial. Lancet 2002;360:7-22.

5 Office for National Statistics. England and Wales; estimated resident population by single year of age and sex; mid-2003 population estimates. Table 3. www.statistics.gov.uk/statbase/ Expodata/Spreadsheets/D8556xls (accessed 11 Oct 2006).

6 Office for National Statistics. Deaths by age, sex and underlying cause, 2002 registrations. Health statistics quarterly 18. www.statistics.gov.uk/StatBase/Expodata/Spreadsheets/ Health statistics quarterly 18. www.

7 Crown copyright. Vital events reference tables. www.gro-scotland.gov.uk/statistics/library/ vitalevents/03reference-tables/index.html (accessed 24 Oct 2006).

8 Prescription Pricing Authority. NHS electronic drug tariff. www.ppa.org.uk/edt/ July_2005/mindex.htm (accessed 11 Oct 2006).

9 HM Treasury. The green book. Appraisal and evaluation in central government. London: HM Treasury, 2003.

10 Efron B, Tibshirani R. An introduction to the bootstrap. New York: Chapman \& Hall, 1993.

11 Kind P. The EuroQoL instrument: an index of health-related quality of life. In: Spilker $\mathrm{B}$, ed. Quality of life and pharmacoeconomics in clinical trials. 2nd ed. Philadelphia: Lippincott-Raven, 1996.

12 Dolan P, Gudex C, Kind P, Williams A. A social tariff for EuroQol: results from a UK general population survey. Report 138. York: Centre for Health Economics, 1995.

13 Joint Formulary Committee. British national formulary, 49. London: British Medical Association and Royal Pharmaceutical Society of Great Britain, 2005.

14 Heart Protection Study Collaborative Group. Effects of cholesterol-lowering with simvastatin on stroke and other major vascular events in 20536 people with cerebrovascular disease or other high-risk conditions. Lancet 2004;363:757-67.

15 Third Joint Task Force of the European and other Societies. Cardiovascular disease prevention in clinical practice (European guidelines on). New Eur J Cardiovasc Prev Rehabil 2003;1(suppl 1):S1-S78.

16 Williams B, Poulter NR, Brown MJ, Davis M, McInnes GT, Potter JF, et al. British Hypertension Society guidelines for hypertension management 2004 (BHS-IV): summary. BMJ 2004;328:634-40.

17 British Cardiac Society, British Hypertension Society, Diabetes UK, HEART UK, Primary Care Cardiovascular Society, Stroke Association. JBS 2: joint British societies' guidelines on prevention of cardiovascular disease in clinical practice. Heart 2005;91(suppl 5):v1-52.

18 National Institute for Health and Clinical Excellence. Statins for the prevention of cardiovascular events. Technology appraisal 94. London: NICE, 2006. www.nice.org.uk/ page.aspx?o $=$ TA094guidance (accessed 11 Oct 2006).

19 Rawlins MD, Culyer AJ. National Institute for Clinical Excellence and its value judgments. BMJ 2004;329:224-7.

20 UK Prospective Diabetes Study Group. Cost effectiveness analysis of improved blood pressure control in hypertensive patients with type 2 diabetes: UKPDS $40 . B M J$ pressure control

21 Thompson PD, Clarkson P, Karas RH. Statin-associated myopathy. JAMA 2003;289:1681-90.

22 Strandberg TE, Pyorala K, Cook TJ, Wilhelmsen L, Faergeman O, Thorgeirsson G, et al. Mortality and incidence of cancer during 10-year follow-up of the Scandinavian simvastatin survival study (4S). Lancet 2004;364:771-7.

(Accepted 22 September 2006)

doi 10.1136/bmj.38993.731725.BE

Correspondence to: hps@ctsu.ox.ac.uk 